\title{
Management of obstructive sleep apnea in edentulous patients: an overview of the literature
}

\author{
David S. P. Heidsieck ${ }^{1}$ Maurits H. T. de Ruiter ${ }^{1}$ - Jan de Lange ${ }^{1}$
}

Received: 4 May 2015 /Revised: 20 October 2015 / Accepted: 28 October 2015 /Published online: 19 November 2015

(C) The Author(s) 2015. This article is published with open access at Springerlink.com

\begin{abstract}
Purpose A high prevalence of obstructive sleep apnea (OSA) is seen in edentulous individuals. Treatment options for edentulous OSA patients however are limited with continuous positive airway pressure therapy (CPAP) remaining the current therapy of choice. As CPAP is associated with high non-adherence rates and oral appliance therapy requiring sufficient dentition, there is a clinical need for effective treatment strategies aimed at edentulous OSA patients. The purpose of this study was to present a thorough overview of the literature regarding (1) the effects of nocturnal denture wearing on OSA, (2) the outcomes of oral appliance therapy, and (3) surgical treatment in edentulous OSA patients.

Methods A computer-assisted literature search was performed in the MEDLINE database on "edentulism" and "obstructive sleep apnea." The search yielded a total of 34 original articles. Results A total of 20 studies were included after exclusion of non-relevant, duplicate, and non-English publications, comprising 4 randomized clinical trials, 12 case reports, and 4 cohort or cross-sectional studies. The outcomes of these studies were addressed in detail concerning nocturnal wearing of dentures, oral appliance therapy, and surgical treatment.

Conclusion Currently, there is no consensus in the literature on the effects of nocturnal wearing of dentures on OSA. Several studies report the successful use of oral appliance therapy, including implant-retained mandibular advancement devices
\end{abstract}

David S. P. Heidsieck

d.s.heidsieck@amc.uva.nl

1 Department of Oral and Maxillofacial Surgery, Suite A1-121, Academic Medical Center, University of Amsterdam, Meibergdreef 9, 1105 AZ Amsterdam, The Netherlands
(MADs), in selected cases of edentulous patients with varying stages of OSA. Little evidence is available regarding outcomes of surgical procedures in edentulous patients. Based on the results of this literature overview, the paucity of effective evidence-based treatment strategies for edentulous OSA patients indicates the further need of clinical studies to improve clinical management.

Keywords Obstructive sleep apnea $\cdot$ Edentulism . Oral appliance therapy $\cdot$ Dentures

\section{Background}

Obstructive sleep apnea (OSA) is a major medical problem, estimated to affect up to $15-30 \%$ of male adults and up to $5-15 \%$ of female adults $[1,2]$. It is characterized by repetitive obstructions of the upper airway during sleep, frequently causing oxygen desaturation. This induces repeating arousals resulting in fragmented sleep and excessive daytime sleepiness. Untreated OSA can lead to a number of complications including cardio- and cerebrovascular disease [3, 4], diabetes mellitus [5], impaired cognitive functions [6], and depression [7]. Additionally, OSA patients are more likely to be involved in motor vehicle crashes [8] and have an increased risk of all-cause mortality compared to individuals without OSA [9].

The underlying pathophysiology of OSA may vary by age, with increased upper airway collapsibility being a common cause in older patients [10]. Previous studies revealed that patients suffering from edentulism are more likely to develop OSA $[11,12]$. The absence of dentition causes a loss of vertical dimension and is associated with morphological changes in the upper airway, decrease of the retropharyngeal space, and decrease in size and tone of the pharyngeal musculature 
$[13,14]$. Despite advances in primary dental care, edentulism remains a common condition in senior adults, with incidence rates between 3 and $80 \%$ depending on the country of residence $[15,16]$.

Current guidelines propose non-surgical treatment options including lifestyle adjustments, position therapy, and (nasally applied) continuous positive airway pressure (CPAP) as firstline therapy. Second in line are treatments with oral appliances or surgical approaches based on the degree of severity and etiology [17]. Edentulism considerably reduces the number of available treatment options, with CPAP generally being the treatment of choice. While the efficacy of CPAP is reliant on patient adherence, non-adherence rates ranging from 29 to $83 \%$ are reported, with many patients rejecting therapy within the first months after initiation. Issues with mask discomfort, nasal dryness or congestion, and difficulty adapting to the pressure have been identified as barriers to patient compliance in CPAP therapy $[18,19]$. Although oral appliance therapy has proven to be an effective non-invasive therapy suitable for patients with mild to moderate OSA, they generally are not indicated for edentate patients as sufficient dentition is required to support and retain the appliance [20]. Petit et al. reported that in up to $34 \%$ of all OSA cases, oral appliance therapy is contraindicated due to dental limitations [21]. Although other therapies including soft tissue surgery or maxillomandibular advancement (MMA) surgery can be considered by clinicians to treat more severe cases of OSA, few evidence is reported in the literature regarding outcomes of such procedures in edentulous OSA patients.

As treatment of edentulous OSA patients can be challenging, all available treatment options should be considered to ensure optimal management and prevent possible life-threatening complications. The present study aims to provide a thorough overview of the literature regarding (1) the effects of nocturnal denture wearing on OSA, (2) the outcomes of oral appliance therapy, and (3) surgical treatment in edentulous OSA patients.

\section{Methods}

A computer-assisted search was performed in the medical database MEDLINE (from January 1966 to March 2015). A combination of the following medical subject headings (MESH) were used in the search: "Sleep Apnea, Obstructive," "Jaw, Edentulous," "Jaw, Edentulous Partially," and "Mouth, Edentulous." Inclusion criteria consisted of studies reporting clinical outcomes on treatment of OSA in either partially or fully edentulous patients. Clinical outcome parameters were considered relevant when comprising results from polysomnography studies (PSG) or standardized sleep-related questionnaires. Excluded were studies not reporting on partially or fully edentulous patients, studies lacking relevant clinical outcomes, review articles, non-original studies, and articles written in other languages than English. Potential relevance of the articles was determined by studying their titles and abstracts. When considered relevant, full texts were obtained and read; other studies were included by screening the reference lists of articles that were found during our original search. Relevant data regarding patient characteristics, treatment, and clinical outcome including age, gender, body mass index (BMI), polysomnography results, and questionnaire results were extracted. Included studies were appraised according to the Oxford Centre for Evidence-Based Medicine levels of evidence (Table 1) [22].

\section{Results}

Our search yielded a total of 34 articles. One study was excluded for being retracted from the respective journal, 1 article reported duplicate findings, and 11 articles did not report clinical outcomes or were non-relevant to the topic. Another study published in a language other than English was excluded. No review articles were identified. The remaining 20 studies, comprising 4 clinical trials, 12 case reports or case series, 3 cross-sectional studies, and 1 cohort study, were included for further examination.

The results and outcomes of all included studies are summarized in Tables 2, 3, and 4 and will be discussed from here on. Table 2 provides an overview of clinical studies investigating the effects of nocturnal wear of dentures on OSA. Studies reporting treatment of edentulous OSA patients with oral appliances are summarized in Table 3. Table 4 summarizes studies reporting surgical treatment of edentulous OSA patients.

\section{Nocturnal use of dentures}

Edentulism is associated with anatomical changes and it is associated with an increased prevalence of OSA [12].

Table 1 Oxford Centre for Evidence-Based Medicine levels of evidence

\begin{tabular}{ll}
\hline Level & Therapy \\
\hline $1 \mathrm{a}$ & Systematic review of randomized controlled trials \\
$1 \mathrm{~b}$ & Individual randomized controlled trial \\
$2 \mathrm{a}$ & Systematic review of cohort studies \\
$2 \mathrm{~b}$ & Individual cohort study \\
$2 \mathrm{c}$ & "Outcomes research" \\
$3 \mathrm{a}$ & Systematic review of case-control studies \\
$3 \mathrm{~b}$ & Individual case-control study \\
4 & Case series (with or without comparison) \\
5 & Expert opinion \\
\hline
\end{tabular}


Dentures are intended to restore this natural anatomy and are being recognized to cause changes in the mandible, tongue, soft tissue, and the pharyngeal airway space [23]. As a result of this, wearing dentures during sleep has been proposed to promote an increase in the retroglossal space and prevent or reduce OSA in edentulous patients [11, 12].

With a study of six edentulous OSA patients, Bucca et al. [11] were the first authors to report the relationship between edentulism, nocturnal denture wearing, and OSA. In patients not wearing their dentures overnight, the authors found a significant worsening of apnea-hypopnea index (AHI) and nocturnal oxygen saturation $\left(\mathrm{SaO}_{2}\right)$ levels, as well as a reduction of the retropharyngeal space. Wearing complete dentures overnight showed to improve both $\mathrm{AHI}$ and $\mathrm{SaO}_{2}$ levels and resulted in an increase in the retropharyngeal space.

Similar findings were found in a larger clinical trial evaluating PSG results and cephalometric exams (CE) of 48 edentulous individuals with complete dentures [12]. In this study, participants were likewise monitored during sleep on two consecutive nights, one night with dentures and one night without. PSG examinations in this study were taken either at home or in-hospital. Almost half of the participants met the criteria for OSA, while the remaining participants did not appear to have any sleep-related breathing disorder. An improvement of AHI and mean and lowest $\mathrm{SaO}_{2}$ was seen in both OSA and non-OSA patients when wearing complete dentures during sleep. These positive effects appeared to be more present in non-OSA patients, suggesting the lack of dentition might play an essential causal role in the AHI increase in these individuals. CE performed in supine position displayed an increase in retropharyngeal space when patients were wearing their dentures. Also, a significant decrease in measured exhaled nitric oxygen (eNO), a marker for inflammation, was found in patients after sleeping with dentures compared to sleeping without dentures. Though the exact role of inflammation in the presence of OSA is still unclear, previous research revealed that pharyngeal inflammation was present in patients with OSA [24, 25].

The studies mentioned above performed by Bucca et al. $[11,12]$ indicate that nocturnal wearing of dentures could be helpful in reducing OSA in edentulous individuals. Other studies however report conflicting results or found positive effects in only the minority of patients. Almeida et al. [26], for example, reported a significant increase in apneic events in patients wearing their dentures overnight. In their clinical trial, 23 OSA patients received two in-laboratory PSG examinations: one with dentures and one night without. Overall, a worsening of AHI was reported in patients wearing their dentures during the night. In patients with mild OSA (AHI 5-15), a significant worsening of AHI was found while wearing dentures overnight, especially when sleeping in supine position. The authors note however that 


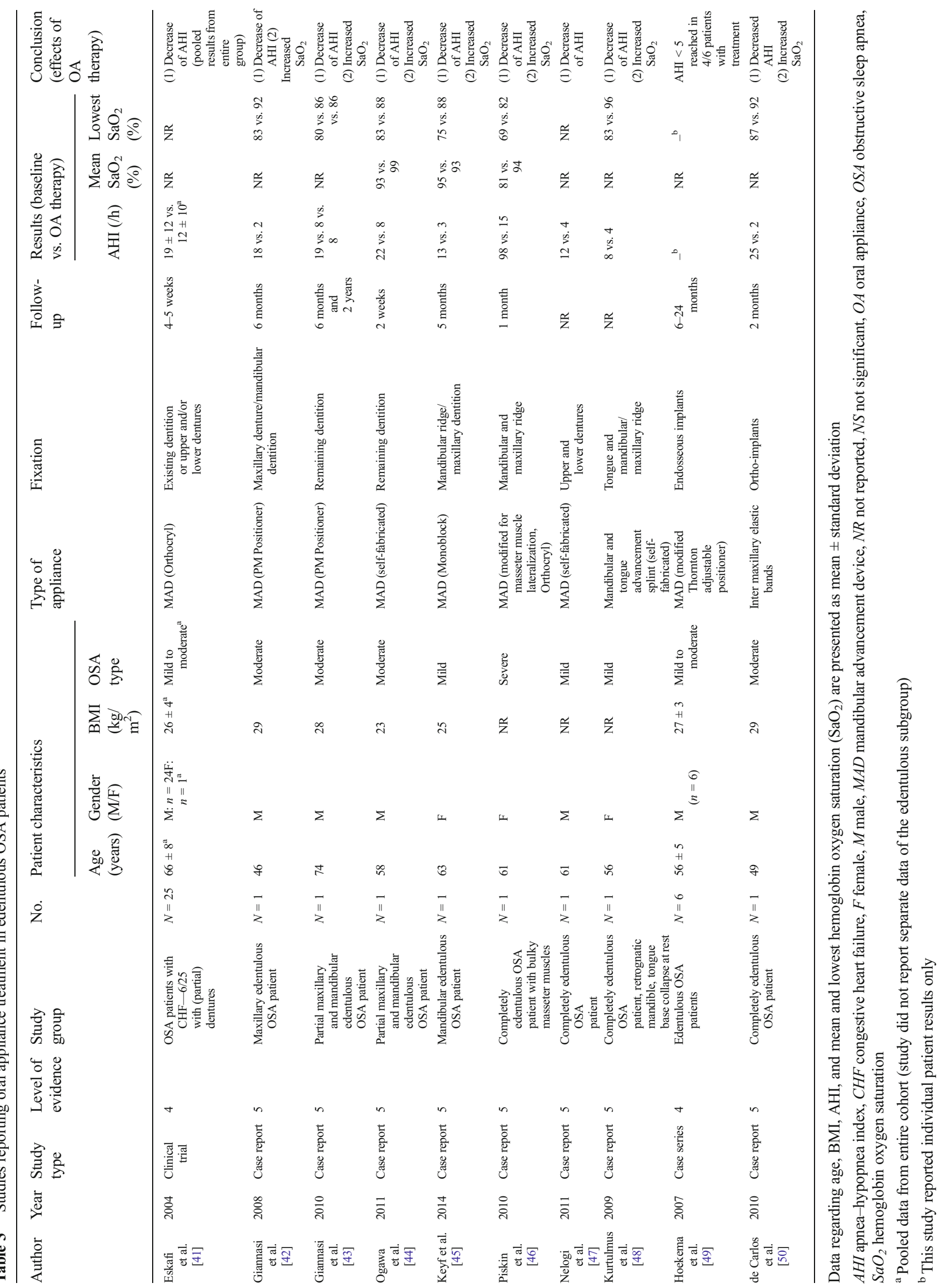




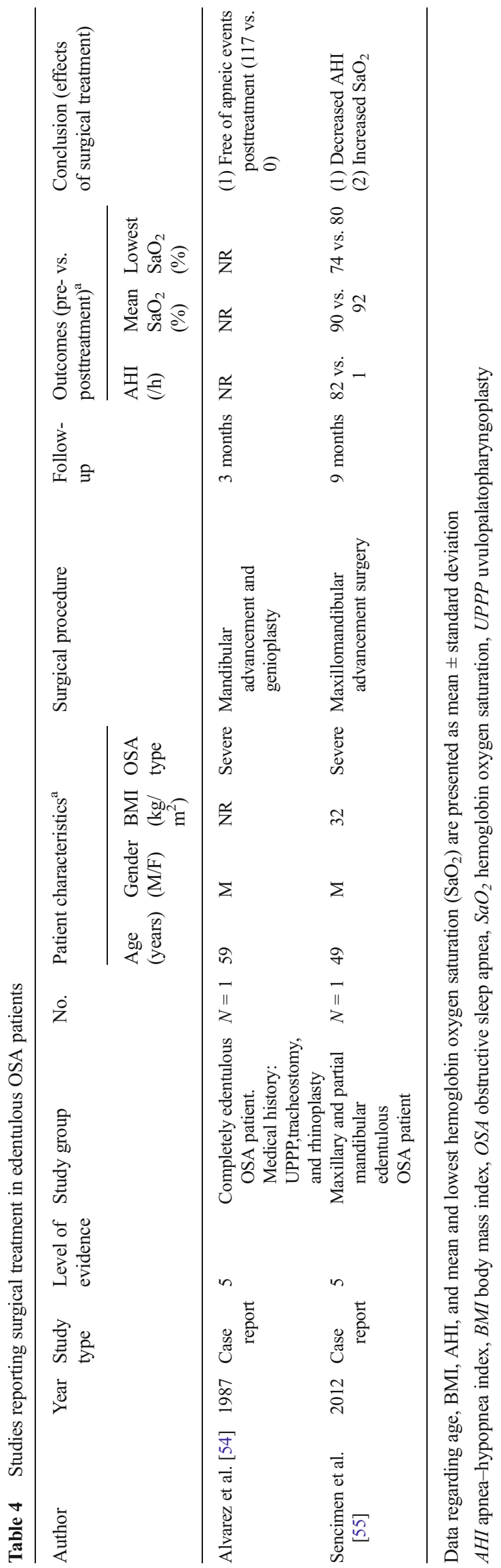

no significant difference was found in patients with moderate (AHI 15-30) or severe (AHI > 30) OSA, with some patients showing an improvement of AHI when wearing dentures overnight. Also, no significant differences were reported on nocturnal $\mathrm{SaO}_{2}$ levels in patients wearing dentures overnight compared to no denture wearing.

Arisaka et al. [27] also reported that nocturnal use of dentures does not assure an improvement of PSG parameters in all patients. In a similar trial with 27 edentulous elderly using portable sleep recording, 19 patients showed to benefit from nocturnal wearing of dentures, while in 8 patients, a worsening of AHI was observed when wearing their dentures overnight.

Several non-experimental studies have examined the relationship between nocturnal wearing of dentures and OSA as well. In a cross-sectional study of 58 elderly using questionnaires and portable sleep recordings, Endeshaw et al. [28] found a relationship between an AHI greater than 15 and denture use, with the majority of patients in their cohort not wearing their dentures overnight. In another cross-sectional study of 173 edentulous elderly, Emami et al. [29] used standardized questionnaires including the Pittsburgh Sleep Quality Index (PSQI), Epworth Sleepiness Scale (ESS), and Karolinska Sleepiness Scale (KSS) to assess the overall sleep quality and daytime sleepiness in elderly. However, no significant difference in sleep quality or daytime sleepiness was found in elderly who preferred to wear their dentures overnight compared to elderly who did not. A cohort study assessed the effects of nocturnal wear of dentures with a follow-up period of 1 year. Again, no significant differences in sleep quality or daytime sleepiness were found [30]. In another cross-sectional study using self-reported enquiries, Tsuda et al. [31] found no evidence that nocturnal denture wearing increased or reduced the risk of sleep disordered breathing.

\section{Oral appliance therapy}

Oral appliance therapy is considered an effective treatment option for patients with mild to moderate OSA and a feasible alternative for patients intolerant to CPAP treatment $[18,32]$. Among these devices, the mandibular advancement device (MAD) is the most commonly used and studied appliance [33]. MADs cover the upper and lower dentition and maintain the mandible in a protruded position during sleep. By enlarging the upper airway and reducing upper airway collapsibility (e.g., by improving upper airway muscle tone), these devices help in sustaining upper airway patency [34, 35]. In awake OSA patients, MAD insertion significantly increases electromyogram (EMG) activity of the genioglossus, geniohyoid, and masseter muscles [36].

Due to a lack of dentition to support and retain a MAD, these devices are considered unsuitable for edentulous OSA patients. If sufficient dentition is available, other 
contraindications to consider include temporomandibular joint (TMJ) dysfunction, severe dental mobility, and periodontal diseases [20, 21]. When MAD treatment is initiated, possible side effects that could hamper compliance include excessive salivation and poor retention on the short term and TMJ pain and myofacial discomfort on the long term [37].

Tongue retaining/reposition devices (TRDs) do not require support from dentition and can be used as an alternative in patients lacking sufficient dentition. TRDs use suction forces to hold the tongue in a forward position without mandibular repositioning and have been reported to have a similar performance to that of MADs [38]. Deane et al. investigated the efficacy of TRDs compared to MAD therapy in a randomized controlled trial and reported a similar effectiveness for both devices in terms of AHI reduction. However, side effects including soft tissue irritation, dryness of mouth, and excessive salivation were reported in the majority of patients treated with a TRD, with MAD therapy showing a substantial higher compliance and patient preference [39]. In a small study comparing three types of oral appliances, Barthlen et al. even reported no improvement of $\mathrm{AHI}$ in patients treated with a TRD [40].

Several case studies report the use of oral appliances in both partial and completely edentulous patients with OSA. Eskafi et al. [41] performed a clinical trial assessing the effects of MAD treatment in OSA patients with stable congestive heart failure (CHF). Six out of 25 participating patients in their study had removable dentures (4 patients with complete dentures, 2 patients with partial dentures) on which a MAD was fixed. Pooled data of all patients showed a significant improvement of AHI compared to pretreatment AHI. No specific analysis on the group of patients wearing dentures however was mentioned. Besides loosening of a fixed partial denture in the upper jaw of one patient after 6 months of treatment, no specific complications regarding the use of the devices in edentulous patients were reported. Overall, 10 patients reported complaints related to MAD use including TMJ pain, soreness of dentition, and tiredness of the jaws. These adverse effects became less intense during the course of the study.

Giannasi et al. [42] applied an adjustable MAD on a partial edentulous OSA patient with a dentate mandible. The patient's pretreatment AHI of 18 decreased to $2 / \mathrm{h}$ posttreatment, and no complaints regarding wearing discomfort were mentioned. In a second case report [43], results were described regarding the use of an adjustable MAD in a partial edentate patient with moderate OSA. In their study, the MAD was fixed to remaining dentition in both jaws, resulting in a significant improvement of nocturnal $\mathrm{AHI}$ and $\mathrm{SaO}_{2}$ levels. A 2-year follow-up showed no deterioration in periodontal and gingival health. Ogawa et al. [44] also reported an improved AHI after MAD treatment of a patient with moderate OSA, missing multiple teeth and harboring severe dental problems. No side effects related to the treatment occurred during the 3-year follow-up. The authors did mention that periodical dental care was required to maintain proper oral health. Keyff et al. [45] used MAD therapy in a patient with mandibular edentulism suffering from mild OSA resulting in improvement of AHI and $\mathrm{SaO}_{2}$ levels. No problems were reported regarding retention or stability of the device.

The use of MAD therapy in a completely edentulous patient with severe OSA refusing CPAP treatment was reported by Piskin et al., resulting in a large improvement of AHI ( 98 vs. $15 / \mathrm{h}$ ) and $\mathrm{SaO}_{2}$ levels [46]. A modified MAD was used to provide mandibular advancement as well as lateral displacement of the patient's bulky masseter muscles, providing more space for the tongue. The authors mentioned a small increase in salivation and discomfort due to full-night usage of the MAD. Nelogi et al. [47] also reported MAD treatment in a completely edentate patient, in this case suffering from mild OSA. An adjusted MAD was fixed on the existing removable lower and upper dentures, resulting in improvement of AHI levels. The authors reported no complaints of discomfort during the 24-week follow-up. Kurtulumus et al. [48] used a selffabricated mandibular and tongue advancement splint in a completely edentulous patient with mild OSA. The authors reported improvement of both AHI levels ( 8 vs. $4 / \mathrm{h}$ ) and nocturnal $\mathrm{SaO}_{2}$. No difficulties regarding stability and usage of the device were reported.

The use of dental implants has been proposed to improve retention, as poor device retention remains a major obstacle of oral appliance treatment in edentulous patients [21, 32]. Hoekema et al. [49] reported a series of 6 edentulous patients with mild to moderate OSA who were treated with endosseous implant-retained MADs. The MADs were fixed to the mandible with a bar construction and clip attachment fixture on four dental implants that were placed in the mandible. The upper part was fixed on the maxillary ridge using suction forces. An improvement of AHI and $\mathrm{SaO}_{2}$ levels was notable in all 5 patients who completed follow-up, and treatment resulted in eliminating the OSA $(\mathrm{AHI}<5)$ in 4 patients. Wearing discomfort was reported in only 2 patients due to excessive pressure of the MAD on the labial mucosa in the maxilla. The authors suggested to resolve such issues of discomfort with additional implant placement in the maxilla. In the single patient that underwent such a secondary treatment, complaints of wearing discomfort were resolved. This pilot study indicates that implant-retained MAD therapy could be a viable treatment option for completely edentulous OSA patients by evading poor retention and wearing discomfort.

A different intraoral approach was suggested by de Carlos et al. [50], involving the use of ortho-implants as an alternative to patients intolerant to CPAP and lacking sufficient dentition. In their case study, orthodontic elastic 
rubbers were anchored to two ortho-implants placed in the maxilla and two implants in the mandible of a completely edentate patient with mild OSA. Wearing of the elastics provided advancement of the mandible and resulted in an AHI drop from 25 to $2 / \mathrm{h}$ as well as improved nocturnal $\mathrm{SaO}_{2}$ levels. No problems occurred during the 8-month follow-up; however, the authors did mention the risk of loss of the ortho-implants due dynamic forces.

\section{Surgical treatment}

Several soft tissue surgical procedures like uvulopalatopharyngoplasty (UPPP) and radiofrequency ablation (RFA) are available for OSA patients, although accompanied by varying success rates $[51,52]$. In patients with severe OSA intolerant to CPAP or in whom oral devices have been found ineffective, maxillomandibular advancement (MMA) surgery has favorable efficacy regarding surgical treatment options [17, 53]. No studies could be identified reporting results of soft tissue surgical procedures in edentate patients. Limited evidence is available regarding treatment and morbidity outcomes of MMA surgery in the edentate patient.

More than two decades ago, Alvarez et al. [54] reported results of a mandibular advancement and genioplasty in an edentulous OSA patient with a medical history of UPPP, temporary tracheostomy, and rhinoplasty. Intermaxillary fixation was performed using transantral K-wire-fixed maxillary dentures and circummandibular wire-fixed mandibular dentures. No peri- or postoperative complications were reported, besides wound dehiscence, which was resolved with removal of one screw in the right ramus. Three months after the MMA procedure, the patient was free of apneic events. The authors however did not report AHI or nocturnal $\mathrm{SaO}_{2}$ values. A more recent case study of Sencimen et al. [55] also reported MMA surgery in a patient with complete maxillary and partial mandibular edentulism suffering from severe OSA. Temporary intermaxillary fixation was performed with wiresecured maxillary and mandibular dentures anchored to the lower residual ridge and palatinal bone with titanium mini screws. Although this patient did not have an atrophic maxilla, no intraoperative complications were reported, nor were there any other complications mentioned. Treatment resulted in an AHI drop from 82 preoperatively to $1 / \mathrm{h}$ postoperatively as well as improved nocturnal $\mathrm{SaO}_{2}$ levels.

\section{Discussion}

Previous studies indicate that edentulous individuals are at high risk of developing OSA [11, 14]. While denture wearing may help restore the natural anatomy in edentulous OSA patients, the effect of overnight denture wearing on OSA remains controversial. Bucca et al. $[11,12]$ reported a significant improvement of PSG parameters in individuals wearing dentures overnight, while Almeida et al. [26] reported a worsening of these parameters after conducting a similar study. Results from Almeida et al. however showed no significant effect in patients having moderate or severe OSA, with some patients even showing an improvement of PSG parameters. To explain these findings, the authors suggested that overnight denture wearing might interrupt activation of the muscles responsible for pulling the mandible up and forward, thereby decreasing the pharyngeal patency. In patients with severe OSA, the ability to maintain appropriate muscle tone may already have been lost. These patients therefore may not experience interruption of muscle activation but receive muscle stimulation from wearing dentures, thus increasing pharyngeal patency. The authors however noted that their results may be limited since a higher average BMI was present in the mild OSA group compared to the moderate to severe OSA group.

Although the variance in the use of portable sleep recordings instead of in-laboratory PSGs among studies might have played a role in the conflicting study results, several studies confirm that ambulant PSGs are a reliable alternative for inlaboratory PSGs [56, 57].

Despite the fact that the majority of edentate patients wear their dentures overnight [58], it is generally not recommended by prosthodontists as it can result in denture stomatitis and traumatic ulcers due to pressure on the soft tissues [59]. Taking into account the potential oral health risks and current absence of conclusive evidence, clinicians treating edentate patients with OSA should be reserved in endorsing patients to wear their dentures at night.

Various treatment options have been proposed to treat OSA, ranging from oral appliance therapy to surgical interventions [17]. Morphological alterations in the edentate patient however may hamper the outcome of or compliance to these interventions when applied in edentulous patients. By addressing the major concern of poor retention due to insufficient dentition, implant-retained MADs appear to be a promising treatment option in patients with mild to moderate OSA. Excessive pressure of the MAD on the maxillary alveolar ridge however was related to wearing discomfort and reduced maximal mandibular advancement [49]. In the pilot study of Hoekema et al., these complaints were resolved by the placement of maxillary dental implants. The increased rate of alveolar bone resorption in edentate patients however should be taken into consideration, as this limits the ability of maxillary implant placement and retention [60]. Similar concerns are present in surgical treatment with orthoimplants [50] as increased bone resorption could compromise treatment success due to implant loss. These risks are also present in other procedures. Although considered a rare complication during MMA surgery in dentate patients, a high incidence of fractures have been described in the 
atrophic edentulous maxilla following LeFort 1 osteotomies. These fractures mostly involved the junction between the horizontal plate of the palatine bone and posterior part of the maxilla [61]. Such complications can eventually lead to aseptic necrosis and surgeons should bare that in mind when considering this intervention [62].

It should be noted that OSA patients in general have an increased risk of postoperative complications including postoperative desaturation, respiratory failure, postoperative cardiac events, and ICU transfers [63]. These general risks should be taken into account when considering surgical treatment, especially in the treatment of elderly patients as they are prone to increased risks of postoperative complications [64].

Unfortunately, there is a lack of clinical trials assessing the application of OSA therapies in edentulous patients. In order to improve clinical management, future studies in the form of randomized controlled trials (RCT) that assess the potential of implant-retained MAD therapy and other inventions are crucial. Adequate treatment is of key importance in an effort to reduce potentially serious complications and improve both the overall health and quality of life of these usually elderly patients $[3,65,66]$.

\section{Conclusion}

Currently, there is no consensus on the effects of nocturnal denture wearing on OSA. The use of modified MADs in edentate patients has been reported successful in selected cases. By avoiding poor retention, implant-retained MADs seem to be a viable treatment alternative to CPAP in the treatment of edentulous OSA patients. Few studies demonstrated the outcomes from surgical treatment in edentulous OSA patients. The overview of the literature presented in this paper demonstrates the paucity of effective evidencebased therapeutic strategies for edentulous OSA patients and the need for further clinical studies to improve clinical management.

\section{Acknowledgments None}

\section{Compliance with ethical standards}

Ethical standards This manuscript does not contain original clinical studies or patient data.

Ethical approval This article does not contain any studies with human participants or animals performed by any of the authors.

Conflict of interest The authors declare that they have no competing interests.

Funding statement This research received no specific grant from any funding agency in the public, commercial, or not-for-profit sectors.
Open Access This article is distributed under the terms of the Creative Commons Attribution 4.0 International License (http:// creativecommons.org/licenses/by/4.0/), which permits unrestricted use, distribution, and reproduction in any medium, provided you give appropriate credit to the original author(s) and the source, provide a link to the Creative Commons license, and indicate if changes were made.

\section{References}

1. Young T, Palta M, Dempsey J, Peppard PE, Nieto FJ, Hla KM (2009) Burden of sleep apnea: rationale, design, and major findings of the Wisconsin Sleep Cohort study. WMJ 108(5):246-249

2. Peppard PE, Young T, Barnet JH, Palta M, Hagen EW, Hla KM (2013) Increased prevalence of sleep-disordered breathing in adults. Am J Epidemiol 177(9):1006-1014

3. Marin JM, Carrizo SJ, Vicente E, Agusti AG (2005) Long-term cardiovascular outcomes in men with obstructive sleep apnoeahypopnoea with or without treatment with continuous positive airway pressure: an observational study. Lancet 365(9464): 1046-1053

4. Yaggi HK, Concato J, Kernan WN, Lichtman JH, Brass LM, Mohsenin V (2005) Obstructive sleep apnea as a risk factor for stroke and death. N Engl J Med 353(19):2034-2041

5. Tasali E, Mokhlesi B, Van Cauter E (2008) Obstructive sleep apnea and type 2 diabetes: interacting epidemics. Chest 133(2):496-506

6. Olaithe M, Bucks RS (2013) Executive dysfunction in OSA before and after treatment: a meta-analysis. Sleep 36(9):1297-1305

7. Gupta MA, Simpson FC (2015) Obstructive sleep apnea and psychiatric disorders: a systematic review. J Clin Sleep Med 11(2): $165-175$

8. George CF (2007) Sleep apnea, alertness, and motor vehicle crashes. Am J Respir Crit Care Med 176(10):954-956

9. Marshall NS, Wong KK, Liu PY, Cullen SR, Knuiman MW, Grunstein RR (2008) Sleep apnea as an independent risk factor for all-cause mortality: the Busselton Health Study. Sleep 31(8): 1079-1085

10. Edwards BA, Wellman A, Sands SA, Owens RL, Eckert DJ, White DP, Malhotra A (2014) Obstructive sleep apnea in older adults is a distinctly different physiological phenotype. Sleep 37(7):1227-1236

11. Bucca C, Carossa S, Pivetti S, Gai V, Rolla G, Preti G (1999) Edentulism and worsening of obstructive sleep apnoea. Lancet 353(9147):121-122

12. Bucca C, Cicolin A, Brussino L, Arienti A, Graziano A, Erovigni F, Pera P, Gai V, Mutani R, Preti G, Rolla G, Carossa S (2006) Tooth loss and obstructive sleep apnoea. Respir Res 7:8

13. Öwall B, Käyser AF, Carlsson GE (1996) Prosthodontics: principles and management strategies. Mosby-Wolfe

14. Gupta P, Thombare R, Pakhan AJ, Singhal S (2011) Cephalometric evaluation of the effect of complete dentures on retropharyngeal space and its effect on spirometric values in altered vertical dimension. ISRN Dent 2011:516969

15. Carlsson GE, Omar R (2010) The future of complete dentures in oral rehabilitation. A critical review. J Oral Rehabil 37(2):143-156

16. Felton DA (2009) Edentulism and comorbid factors. J Prosthodont 18(2):88-96

17. Epstein LJ, Kristo D, Strollo Jr PJ, Friedman N, Malhotra A, Patil SP, Ramar K, Rogers R, Schwab RJ, Weaver EM, Weinstein MD (2009) Clinical guideline for the evaluation, management and longterm care of obstructive sleep apnea in adults. J Clin Sleep Med 5(3):263-276 
18. Rosenberg R, Doghramji P (2009) Optimal treatment of obstructive sleep apnea and excessive sleepiness. Adv Ther 26(3):295-312

19. Zozula R, Rosen R (2001) Compliance with continuous positive airway pressure therapy: assessing and improving treatment outcomes. Curr Opin Pulm Med 7(6):391-398

20. Ferguson KA (2003) The role of oral appliance therapy in the treatment of obstructive sleep apnea. Clin Chest Med 24(2):355-364

21. Petit FX, Pepin JL, Bettega G, Sadek H, Raphael B, Levy P (2002) Mandibular advancement devices: rate of contraindications in 100 consecutive obstructive sleep apnea patients. Am J Respir Crit Care Med 166(3):274-278

22. OCEBM Levels of Evidence Working Group. The Oxford 2011 levels of evidence. Oxford Centre for Evidence-Based Medicine. http://www.cebmnet/indexaspx?o=1025

23. Erovigni F, Graziano A, Ceruti P, Gassino G, De Lillo A, Carossa S (2005) Cephalometric evaluation of the upper airway in patients with complete dentures. Minerva Stomatol 54(5):293-301

24. Olopade CO, Christon JA, Zakkar M, Hua C, Swedler WI, Scheff PA, Rubinstein I (1997) Exhaled pentane and nitric oxide levels in patients with obstructive sleep apnea. Chest 111(6):1500-1504

25. Sekosan M, Zakkar M, Wenig BL, Olopade CO, Rubinstein I (1996) Inflammation in the uvula mucosa of patients with obstructive sleep apnea. Laryngoscope 106(8):1018-1020

26. Almeida FR, Furuyama RJ, Chaccur DC, Lowe AA, Chen H, Bittencourt LR, Frigeiro ML, Tsuda H (2012) Complete denture wear during sleep in elderly sleep apnea patients - a preliminary study. Sleep Breath 16(3):855-863

27. Arisaka H, Sakuraba S, Tamaki K, Watanabe T, Takeda J, Yoshida K (2009) Effects of wearing complete dentures during sleep on the apnea-hypopnea index. Int J Prosthodont 22(2):173-177

28. Endeshaw YW, Katz S, Ouslander JG, Bliwise DL (2004) Association of denture use with sleep-disordered breathing among older adults. J Public Health Dent 64(3):181-183

29. Emami E, Lavigne G, de Grandmont P, Rompre PH, Feine JS (2012) Perceived sleep quality among edentulous elders. Gerodontology 29(2):e128-e134

30. Emami E, Salah MH, Rompre P, Huynh N, Beauchamp A, Feine JS (2013) The nocturnal use of complete dentures and sleep stability in edentulous elders. J Dent 41(8):703-709

31. Tsuda H, Almeida FR, Walton JN, Lowe AA (2010) Questionnaire-based study on sleep-disordered breathing among edentulous subjects in a university oral health center. Int J Prosthodont 23(6):503-506

32. Hoekema A, Stegenga B, De Bont LG (2004) Efficacy and comorbidity of oral appliances in the treatment of obstructive sleep apnea-hypopnea: a systematic review. Crit Rev Oral Biol Med 15(3):137-155

33. Jauhar S, Lyons MF, Banham SW, Cameron DA, Orchardson R (2008) Ten-year follow-up of mandibular advancement devices for the management of snoring and sleep apnea. J Prosthet Dent 99(4):314-321

34. Ferguson KA, Cartwright R, Rogers R, Schmidt-Nowara W (2006) Oral appliances for snoring and obstructive sleep apnea: a review. Sleep 29(2):244-262

35. Chan AS, Lee RW, Cistulli PA (2007) Dental appliance treatment for obstructive sleep apnea. Chest 132(2):693-699

36. Johal A, Gill G, Ferman A, McLaughlin K (2007) The effect of mandibular advancement appliances on awake upper airway and masticatory muscle activity in patients with obstructive sleep apnoea. Clin Physiol Funct Imaging 27(1):47-53

37. Cistulli PA, Gotsopoulos H, Marklund M, Lowe AA (2004) Treatment of snoring and obstructive sleep apnea with mandibular repositioning appliances. Sleep Med Rev 8(6):443-457

38. Lazard DS, Blumen M, Levy P, Chauvin P, Fragny D, Buchet I, Chabolle F (2009) The tongue-retaining device: efficacy and side effects in obstructive sleep apnea syndrome. J Clin Sleep Med 5(5): $431-438$

39. Deane SA, Cistulli PA, Ng AT, Zeng B, Petocz P, Darendeliler MA (2009) Comparison of mandibular advancement splint and tongue stabilizing device in obstructive sleep apnea: a randomized controlled trial. Sleep 32(5):648-653

40. Barthlen GM, Brown LK, Wiland MR, Sadeh JS, Patwari J, Zimmerman M (2000) Comparison of three oral appliances for treatment of severe obstructive sleep apnea syndrome. Sleep Med 1(4):299-305

41. Eskafi M, Ekberg E, Cline C, Israelsson B, Nilner M (2004) Use of a mandibular advancement device in patients with congestive heart failure and sleep apnoea. Gerodontology 21(2):100-107

42. Giannasi LC, Magini M, de Oliveira CS, de Oliveira LV (2008) Treatment of obstructive sleep apnea using an adjustable mandibular repositioning appliance fitted to a total prosthesis in a maxillary edentulous patient. Sleep Breath 12(1):91-95

43. Giannasi LC, Magini M, Costa MS, Oliveira CS, Oliveira LV (2010) Oral appliance treatment for obstructive sleep apnea in a partly edentulous patient. Am J Orthod Dentofac Orthop 137(4):548-551

44. Ogawa T, Ito T, Cardoso MV, Kawata T, Sasaki K (2009) Treatment using oral appliance for obstructive sleep apnea syndrome patients with multiple missing teeth. Sleep Med 10(7):809-811

45. Keyf F, Ciftci B, Firat Guven S (2014) Management of obstructive sleep apnea in an edentulous lower jaw patient with a mandibular advancement device. Case Rep Dent 2014:436904

46. Piskin B, Sentut F, Sevketbeyoglu H, Avsever H, Gunduz K, Kose M, Oguz D, Uyar S (2010) Efficacy of a modified mandibular advancement device for a totally edentulous patient with severe obstructive sleep apnea. Sleep Breath 14(1):81-85

47. Nelogi S, Porwal A, Naveen H (2011) Modified mandibular advancement appliance for an edentulous obstructive sleep apnea patient: a clinical report. J Prosthodont Res 55(3):179-183

48. Kurtulmus H, Cotert HS (2009) Management of obstructive sleep apnea with a mandibular and tongue advancement splint (MTAS) in a completely edentulous patient. A clinical report. J Prosthodont 18(4):348-352

49. Hoekema A, de Vries F, Heydenrijk K, Stegenga B (2007) Implantretained oral appliances: a novel treatment for edentulous patients with obstructive sleep apnea-hypopnea syndrome. Clin Oral Implants Res 18(3):383-387

50. de Carlos F, Cobo J, Fernandez Mondragon MP, Alvarez Suarez A, Calvo Blanco J (2010) Orthoimplants: an alternative treatment for SAHS? Sleep Breath 14(2):171-174

51. Khan A, Ramar K, Maddirala S, Friedman O, Pallanch JF, Olson EJ (2009) Uvulopalatopharyngoplasty in the management of obstructive sleep apnea: the mayo clinic experience. Mayo Clin Proc 84(9): 795-800

52. Brown DJ, Kerr P, Kryger M (2001) Radiofrequency tissue reduction of the palate in patients with moderate sleep-disordered breathing. J Otolaryngol 30(4):193-198

53. Holty JE, Guilleminault C (2010) Maxillomandibular advancement for the treatment of obstructive sleep apnea: a systematic review and meta-analysis. Sleep Med Rev 14(5):287-297

54. Alvarez CM, Lessin ME, Gross PD (1987) Mandibular advancement combined with horizontal advancement genioplasty for the treatment of obstructive sleep apnea in an edentulous patient. A case report. Oral Surg Oral Med Oral Pathol 64(4):402-406

55. Sencimen M, Bayar GR, Akcam T, Altug HA, Altug H, Gulses A, Ozkan A (2012) Management of obstructive sleep apnea by maxillomandibular advancement surgery in an edentulous patient. J Craniofac Surg 23(6):e582-e585

56. Redline S, Tosteson T, Boucher MA, Millman RP (1991) Measurement of sleep-related breathing disturbances in epidemiologic studies. Assessment of the validity and reproducibility of a portable monitoring device. Chest 100(5):1281-1286 
57. Emsellem HA, Corson WA, Rappaport BA, Hackett S, Smith LG, Hausfeld JN (1990) Verification of sleep apnea using a portable sleep apnea screening device. South Med J 83(7): $748-752$

58. de Castellucci BL, Ferreira MR, de Carvalho Calabrich CF, Viana AC, de Lemos MC, Lauria RA (2008) Edentulous patients' knowledge of dental hygiene and care of prostheses. Gerodontology 25(2):99-106

59. Marcus PA, Joshi A, Jones JA, Morgano SM (1996) Complete edentulism and denture use for elders in New England. J Prosthet Dent 76(3):260-266

60. Bianchi A, Sanfilippo F (2002) Osteoporosis: the effect on mandibular bone resorption and therapeutic possibilities by means of implant prostheses. Int J Periodontics Restorative Dent 22(3):231-239

61. Li KK, Stephens W (1996) Fractures of the atrophic, edentulous maxilla during Le Fort I osteotomy. Int J Oral Maxillofac Surg 25(6):430-432
62. Lanigan DT, Hey JH, West RA (1990) Aseptic necrosis following maxillary osteotomies: report of 36 cases. J Oral Maxillofac Surg 48(2):142-156

63. Kaw R, Chung F, Pasupuleti V, Mehta J, Gay PC, Hernandez AV (2012) Meta-analysis of the association between obstructive sleep apnoea and postoperative outcome. Br J Anaesth 109(6):897-906

64. Leung JM, Dzankic S (2001) Relative importance of preoperative health status versus intraoperative factors in predicting postoperative adverse outcomes in geriatric surgical patients. J Am Geriatr Soc 49(8):1080-1085

65. Punjabi NM, Caffo BS, Goodwin JL, Gottlieb DJ, Newman AB, O'Connor GT, Rapoport DM, Redline S, Resnick HE, Robbins JA, Shahar E, Unruh ML, Samet JM (2009) Sleep-disordered breathing and mortality: a prospective cohort study. PLoS Med 6(8): e1000132

66. Moyer CA, Sonnad SS, Garetz SL, Helman JI, Chervin RD (2001) Quality of life in obstructive sleep apnea: a systematic review of the literature. Sleep Med 2(6):477-491 